\title{
ACEF performed better than other risk scores in non-ST-elevation acute coronary syndrome during long term follow-up
}

\author{
Ivica Kristić ${ }^{1 \dagger}$, Nikola Crnčević ${ }^{1}$, Frane Runjić ${ }^{1}$, Vesna Čapkun², Ozren Polašek ${ }^{3}$, Andrija Matetic ${ }^{1}$ \\ and Mislav Vrsalovic ${ }^{4,5^{*}+}$ (B)
}

\begin{abstract}
Background: Risk stratification of patients with non-ST-elevation acute coronary syndrome (NSTE-ACS) is an important clinical method, but long-term studies on patients subjected to all-treatment strategies are lacking. Therefore, the aim was to compare several established risk scores in the all-treatment NSTE-ACS cohort during long-term follow-up.

Methods: Consecutive patients $(n=276)$ with NSTE-ACS undergoing coronary angiography were recruited between September 2012 and May 2015. Six risk scores for all patients were calculated, namely GRACE 2.0, ACEF, SYNTAX, Clinical SYNTAX, SYNTAX II PCI and SYNTAX II CABG. The primary end-point was Major Adverse Cardiovascular Events (MACE) which was a composite of cardiac death, nonfatal myocardial infarction, ischemic stroke or urgent coronary revascularization.

Results: During a median follow-up of 33 months, 64 MACE outcomes were recorded (23.2\%). There was no difference between risk score categories, except in the highest risk group of ACEF and SYNTAX II PCI scores which exhibited significantly more MACE $(51.6 \%, N=33$ and $45.3 \%, N=29, P=0.024$, respectively). In the multivariate Cox regression analysis of individual variables, only age and atrial fibrillation were significant predictors for MACE (HR 1.03, 95\% Cl $1.00-1.05, P=0.023$ and HR 2.02, 95\% Cl 1.04-3.89, $P=0.037$, respectively). Furthermore, multivariate analysis of the risk scores showed significant prediction of MACE only with ACEF score (HR 2.16, 95\% Cl 1.36-3.44, $P=0.001)$. The overall performance of GRACE, SYNTAX, Clinical SYNTAX and SYNTAX II CABG was poor with AUC values of 0.596, 0.507, 0.530 and 0.582 , respectively, while ACEF and SYNTAX II PCI showed the best absolute AUC values for MACE (0.630 and 0.626 , respectively).
\end{abstract}

Conclusions: ACEF risk score showed better discrimination than other risk scores in NSTE-ACS patients undergoing all-treatment strategies over long-term follow-up and it could represent a fast and user-friendly tool to stratify NSTEACS patients.

Keywords: Non-ST-elevation acute coronary syndrome, Risk scores, All-treatment strategies

*Correspondence: mislav.vrsalovic@gmail.com

${ }^{\dagger}$ Ivica Kristić and Mislav Vrsalovic have contributed equally_-joint first authors

${ }^{4}$ Department of Cardiology, Sestre Milosrdnice University Hospital Center, Vinogradska cesta 29, 10000 Zagreb, Croatia

Full list of author information is available at the end of the article

\section{Background}

Non-ST-elevation acute coronary syndrome (NSTEACS) is one of the leading causes of cardiovascular morbidity and mortality, with a rising prevalence in the last decades $[1,2]$. It is comprised out of two closely interconnected clinical entities, namely non-ST-segment elevation myocardial infarction (NSTEMI) and unstable 
angina (UA) $[3,4]$. Improved preventive measures and higher sensitivity of diagnostic methods have led to a decreased incidence of ST-segment elevation myocardial infarction (STEMI) with a relative increase of NSTE-ACS events in the total cohort of acute coronary syndrome (ACS) patients [1].

However, ACS represents a heterogeneous syndrome with significantly different outcomes among its subgroups and subpopulations $[5,6]$. While short-term outcomes are worse in STEMI patients due to a higher rate of in-hospital mortality, NSTE-ACS patients generally exhibit worse long-term adverse outcomes [6]. Furthermore, subgroups of NSTE-ACS (NSTEMI and UA) also differ in long-term prognosis [5], and the appropriate management of these patients still represents a subject of debate [7]. Therefore, the risk stratification of these patients helps to establish the most appropriate therapeutic strategy with short-term and long-term prognostic implications [3, 4]. Recent studies and guidelines advocate that specific high-risk subgroups may benefit from an aggressive therapeutic approach in NSTE-ACS $[3,4]$.

According to the current guidelines, quantitative risk assessment using a clinical risk score Global Registry for Acute Coronary Events (GRACE) may be considered for the prognostic estimation of NSTE-ACS patients [4, 8, 9], while other risk scores like Age, Creatinine, Ejection Fraction (ACEF), The Synergy Between Percutaneous Coronary Intervention with TAXUS and Cardiac Surgery (SYNTAX), Clinical SYNTAX and SYNTAX II score have been previously investigated in similar clinical settings as well [10-14]. However, comprehensive studies comparing the performance of the aforementioned risk scores in NSTE-ACS patients with long-term follow-up are lacking. Therefore, the aim was to compare the longterm discrimination and calibration of several clinical and angiographic risk scores, namely GRACE 2.0, ACEF, SYNTAX, Clinical SYNTAX, SYNTAX II for percutaneous coronary intervention (PCI) and SYNTAX II for coronary artery bypass grafting (CABG), in the NSTE-ACS cohort undergoing all treatment strategies including PCI, CABG or conservative management.

\section{Methods}

\section{Ethical and institutional considerations}

All the proceedings and clinical research were performed in accordance with the ethical standards and amendments of the Declaration of Helsinki. The study protocol was approved by the Ethical Committee of the University Hospital of Split, Croatia (No. 2181-147-01/06). All the participants included in the study provided formal written informed consent for coronary angiography and informed verbal consent for the use of relevant medical data, which is in accordance with the Approval of the Ethical committee of the University Hospital of Split and the Declaration of Helsinki. All participants were informed about the goal and course of this study.

\section{Study design}

This was a single-centre observational prospective study. All patients with NSTE-ACS undergoing a coronary angiography at the University Hospital of Split between September 2012 and May 2015 were considered eligible. Patients with active malignant disease and a history of CABG were excluded (Additional file 1: Fig. S1). All patients, including those receiving a conservative treatment, underwent coronary angiography. Baseline characteristics were obtained from electronic health records. The diagnosis of NSTE-ACS (UA and NSTEMI) was established according to the competent international guidelines [3, 4]. The reasons for loss of follow-up were inability to contact a patient, or patient refusal of further follow-up. All patients were followed up through scheduled clinical visits or telephone interviews firstly 3 months after the index event and thereafter at a 12-month interval, with a final contact in May 2017.

\section{Laboratory analysis}

Blood samples were collected from all participants included in the study. These were used to measure cardiac troponin I, with a threshold for positivity of $0.033 \mathrm{ng} / \mathrm{ml}$ and other usual biochemical parameters. All laboratory analyses were done in the same biochemical laboratory and measured by standard laboratory methods. Glomerular filtration rate (eGFR) was estimated using a Cockcroft-Gault formula.

\section{Treatment strategies}

Coronary angiography was primarily performed over radial access. After a diagnostic coronary angiogram was performed, patients were treated with PCI, CABG or conservatively based on the heart team decision and patient preferences. All patients were treated with a tailored treatment plan, which included dual antiplatelet therapy, in line with the current guidelines [4].

\section{Outcomes}

A key primary endpoint included Major Adverse Cardiac Event (MACE), which was a composite of cardiac death, nonfatal myocardial infarction (MI), ischemic stroke or urgent coronary revascularization. All deaths were considered to be of cardiac origin unless sufficient evidence indicated a non-cardiac cause of death. Nonfatal MI was defined as a recurrent MI with or without ST-elevation or UA. UA was included among MI events only if there was an angiographic confirmation of an unstable lesion and a 
subsequent revascularization. Urgent coronary revascularization was defined as urgent intervention, percutaneous or surgical, due to highly symptomatic stable angina. All outcomes were evaluated by a team of experienced cardiologists (I.K., F.R. and M.V.).

\section{Clinical and risk assessment}

Individual risk was determined for all participants included in the study. Risk assessment was conducted by six established risk scores: GRACE 2.0, ACEF, SYNTAX, Clinical SYNTAX, SYNTAX II for PCI and SYNTAX II for $C A B G$. Necessary information was extracted from patient medical records, electrocardiograms, laboratory analysis, and angiographic data during initial hospitalization. Anthropometric data were collected according to the standard methods.

The GRACE score was calculated using an online calculator version 2.0 [8]. The three-variable $A C E F$ model was calculated according to the following formula: age (years)/left ventricular ejection fraction (percentage) +1 (if serum creatinine $>176 \mu \mathrm{mol} / \mathrm{L}$ [ $>2 \mathrm{mg} / \mathrm{dL}]$ ) [15]. The SYNTAX score was computed from the baseline coronary angiogram, as previously described, by two experienced interventional cardiologists (I.K. and N.C.). In case of disagreement, a third cardiologist re-evaluated coronary angiogram (M.V.) [11]. The Clinical SYNTAX score was calculated by multiplying the values of $A C E F$ and the SYNTAX score [13]. The SYNTAX II score was calculated using the online calculator, as previously described [16]. The overview of the used risk scores is presented in the Additional file 2: Table S1 and S2.

Patients were stratified in different groups according to the risk score values. The following thresholds were used-GRACE score: $<88.0, \quad 8.0-118.0,>119.0$; $A C E F$ score (tertiles): $<1.0,1.0-1.24,>1.24 ;$ SYNTAX score: $\leq 22$, 23-32, $\geq 33$; Clinical SYNTAX score (tertiles): $<10.42,10.42-23.9,>23.9 ;$ SYNTAX score II for PCI (tertiles): $<22.7,22.7-31.6,>31.6$; SYNTAX score II for $C A B G$ (tertiles): $<20.6,20.6-30.8,>30.8$. Cut-off values for GRACE score and SYNTAX score were set according to previously established criteria for 6-month postdischarge mortality and complexity of coronary disease, respectively $[11,17]$. In addition, a novel model has been constructed that encompassed three independent predictor variables from the multivariate analysis: $A C E F$, female gender and atrial fibrillation, and was compared with other risk scores.

\section{Statistical analysis}

Statistical analysis was conducted according to standard statistical methods. The normality of data distribution was assessed by the Kolmogorov-Smirnov test. Continuous data were presented as mean \pm standard deviation
(SD) or as median (interquartile range, IQR). Student's T-test or Mann-Whitney U test were used for continuous data analysis according to parametric or non-parametric distribution, respectively. Categorical variables were expressed as numbers and percentages and analysed using the Chi-squared test. The accuracy of each variable in predicting MACE was tested using receiver operating characteristic (ROC), with a calculation of area under the curve (AUC). The reported $P$ values represent the significance relative to the non-informative ROC curve $(\mathrm{AUC}=0.5)$ and were tested using a SPSS algorithm based on methodology of Hanley and McNeil [18]. The cumulative incidence of MACE was estimated using the Kaplan-Meier approach, and significance was assessed using the Mantel-Cox log-rank test. Cox logistic regression analysis was performed to determine the predictors of MACE in the univariate and the multivariate model. A separate multivariate model of the individual variables and risk scores have been conducted to avoid multicollinearity. A multivariate analysis of individual variables contained all variables with a $P<0.1$ at univariate analysis, while the multivariate analysis of risk scores retained only ACEF, GRACE and SYNTAX II PCI score. The multivariate analysis tested the 10-unit change for GRACE and SYNTAX II PCI scores. The SYNTAX, Clinical SYNTAX and SYNTAX II CABG scores have been excluded from the multivariate analysis to avoid multicollinearity. A stepwise forward algorithm (with a removal criterion set to $P<0.1$ ) was used in the multivariate analysis. The results of the risk analyses are provided as hazard ratio (HR) and 95\% confidence intervals (95\% CI) which corresponds to a 1-unit increase/decrease of each score on a continuous scale. Furthermore, additional HR for all scores (except $A C E F$ ) have been presented from the univariate analysis which correspond to a 10-unit increase/ decrease of each score. Calibration, a measure of the agreement between observed and predicted outcomes, was assessed by the Hosmer-Lemeshow goodness-of-fit test. A two-sided $P$-value of $<0.05$ was considered significant. Statistical data analysis was carried out using a Statistical Package for the Social Sciences (SPSS) software (IBM Corp, NY, USA; version 20).

\section{Results}

A total of 300 patients were initially enrolled in the study protocol, of which 276 completed follow-up. The study population was mostly comprised of older adult male patients $(64.3 \pm 11.4$ years and $74.3 \%, \mathrm{~N}=205$, respectively). The femoral access was used in only $7.4 \%$ patients all others were treated via radial approach. The median follow-up period was 35 months (IQR 26-42 months). Of the 276 patients who completed follow-up, MACE occurred in 64 patients (23.2\%), including 16 cardiac 
deaths (5.8\%), $10 \mathrm{MI}$ (3.6\%), 4 ischemic strokes (1.4\%), and 34 urgent coronary revascularizations (12.3\%). Patients who developed MACE were significantly older and showed a higher prevalence of female patients and atrial fibrillation, but exhibited lower values of BMI, haemoglobin, eGFR and left ventricular ejection fraction $(P<0.05)$. There was no statistically significant difference in other baseline and laboratory parameters (Table 1).

There was no statistically significant difference in treatment strategies, features of the coronary angiogram, discharge therapy and risk scores between the different subgroups, except in the values of $A C E F, S Y N$ TAX II PCI and SYNTAX II CABG score which were significantly higher in patients who developed MACE $(P<0.05)$ (Table 2). Furthermore, there was no significant difference in MACE occurrence among the different NSTE-ACS risk categories, except among the $A C E F$ and SYNTAX II PCI risk categories in which MACE occurred significantly more often in the group with the highest values $(51.6 \%, \mathrm{~N}=33$ and $45.3 \%, \mathrm{~N}=29, P=0.020$, respectively) (Additional file 2: Table S3). Consistently, the binomial logistic regression model revealed that patients from the highest ACEF and SYNTAX II PCI group have significantly higher odds of MACE (OR 2.76, 95\% CI 1.55-4.91, $P=0.001$; and OR $1.96,95 \%$ CI $1.10-3.48$, $P=0.022)$ in comparison to lower tertiles, while there was no statistically significant difference between risk categories for other risk scores.

Among individual variables, age, BMI, eGFR, female gender and atrial fibrillation, were significantly associated with an increased incidence of MACE in univariate analysis $(P<0.05)$, but the only significant independent predictors for MACE in the following multivariate Cox regression analysis proved to be age (HR 1.03, 95\% CI $1.00-1.05, P=0.023$ ) and atrial fibrillation (HR 2.02, 95\% CI $1.04-3.89, P=0.037)$. Furthermore, in the multivariate

Table 1 Comparison of baseline anthropometric, laboratory and echocardiographic parameters of study participants

\begin{tabular}{|c|c|c|c|c|}
\hline \multirow[t]{2}{*}{ Variables } & \multicolumn{2}{|l|}{ MACE } & \multirow[t]{2}{*}{ Total $(n=276)$} & \multirow[t]{2}{*}{$P$ value } \\
\hline & No $(n=212)$ & Yes $(n=64)$ & & \\
\hline Age (years) & $63.2 \pm 10.9$ & $68.0 \pm 11.1$ & $64.3 \pm 11.4$ & $0.002^{*}$ \\
\hline Female gender & $47(22.2)$ & $24(37.5)$ & $71(25.7)$ & $0.014^{\dagger}$ \\
\hline $\operatorname{BMI}\left(\mathrm{kg} / \mathrm{m}^{2}\right)$ & $27.8(25.8-30.9)$ & $26.6(24.4-29.8)$ & $27.8(25.4-30.7)$ & $0.026^{\ddagger}$ \\
\hline NSTE-ACS subtype & & & & $0.733^{+}$ \\
\hline NSTEMI & $175(82.5)$ & $54(84.4)$ & $229(83.0)$ & \\
\hline UA & $37(17.5)$ & $10(15.6)$ & $47(17.0)$ & \\
\hline Arterial hypertension & $131(61.8)$ & $46(71.9)$ & $177(64.1)$ & $0.140^{\dagger}$ \\
\hline Diabetes mellitus & $60(28.3)$ & $23(35.9)$ & $83(30.1)$ & $0.110^{\dagger}$ \\
\hline Hypercholesterolemia & $106(50.0)$ & $32(50.0)$ & $138(50.0)$ & $0.999^{\dagger}$ \\
\hline Family history of CVD & $76(35.8)$ & $25(39.1)$ & $101(36.6)$ & $0.640^{\dagger}$ \\
\hline Active smoking & $62(29.2)$ & $14(21.9)$ & $76(27.5)$ & $0.260^{+}$ \\
\hline Atrial fibrillation & $15(7.1)$ & $11(17.2)$ & $26(9.4)$ & $0.015^{\dagger}$ \\
\hline Prior CAD & $8(3.8)$ & $4(6.3)$ & $12(4.3)$ & $0.395^{\dagger}$ \\
\hline Prior MI & $19(9.0)$ & $11(17.2)$ & $30(10.9)$ & $0.066^{\dagger}$ \\
\hline Prior $\mathrm{PCl}$ & $17(8.0)$ & $6(9.4)$ & $23(8.3)$ & $0.731^{+}$ \\
\hline PAD & $20(9.5)$ & $9(14.1)$ & $29(10.5)$ & $0.432^{\dagger}$ \\
\hline COPD & $26(12.2)$ & $7(10.9)$ & $33(12.0)$ & $0.920^{\dagger}$ \\
\hline Killip class > 1 & $20(9.0)$ & $8(12.5)$ & $28(9.8)$ & $0.692^{\dagger}$ \\
\hline $\mathrm{Hgb}(\mathrm{g} / \mathrm{L})$ & $143.0(134.0-153.0)$ & $137.0(123.3-149.0)$ & $141.2(133.0-152.0)$ & $0.010^{\ddagger}$ \\
\hline Glucose (mmol/L) & $7.1(6.0-9.9)$ & $7.4(6.1-9.6)$ & $7.1(6.0-9.7)$ & $0.672^{\ddagger}$ \\
\hline $\mathrm{CRP}(\mathrm{mmol} / \mathrm{L})$ & $5.1(2.0-10.5)$ & $8.6(1.5-29.3)$ & $5.7(1.7-12.9)$ & $0.128^{\ddagger}$ \\
\hline eGFR (ml/min) & $91.8 \pm 27.1$ & $81.9 \pm 28.3$ & $89.5 \pm 27.7$ & $0.017^{*}$ \\
\hline $\operatorname{LVEF}(\%)$ & $59.0(55.0-65.0)$ & $55.0(51.3-62.0)$ & $58.0(55.0-65.0)$ & $0.046^{\ddagger}$ \\
\hline Follow-up (months) & $36.1(29.5-43.8)$ & $32.6(26.5-43.5)$ & $35.1(29.3-44.0)$ & $0.089^{\ddagger}$ \\
\hline
\end{tabular}

Data are expressed as mean $\pm S D$, number (percent) or median (interquartile range)

BMI_-body mass index; CAD—coronary arterial disease; COPD—chronic obstructive pulmonary disease; CRP—C-reactive peptide; CVD—cardiovascular disease; eGFR —estimated glomerular filtration rate; Hgb—hemoglobin; LVEF—left ventricular ejection fraction; MACE—-major adverse cardiovascular events; MI—myocardial infarction; NSTE-ACS—non-ST elevation acute coronary syndrome; NSTEMI—non ST segment elevation myocardial infarction; UA—unstable angina; PAD—peripheral arterial disease; $\mathrm{PCl}$ - percutaneous coronary intervention

*Student's T test; ${ }^{\dagger}$ Chi-square test; ${ }^{\ddagger}$ Mann-Whitney U test 
Table 2 Comparison of therapeutic and angiographic characteristics of study participants

\begin{tabular}{|c|c|c|c|c|}
\hline \multirow[t]{2}{*}{ Variables } & \multicolumn{2}{|l|}{ MACE } & \multirow[t]{2}{*}{ Total $(n=276)$} & \multirow[t]{2}{*}{$p$ value } \\
\hline & No $(n=212)$ & Yes $(n=64)$ & & \\
\hline Treatments & & & & $0.071^{\dagger}$ \\
\hline Conservative & $43(20.3)$ & $20(31.3)$ & $63(22.8)$ & \\
\hline $\mathrm{PCl}$ & $83(39.2)$ & $27(42.2)$ & $110(39.9)$ & \\
\hline CABG & $86(40.6)$ & $17(26.6)$ & $103(37.3)$ & \\
\hline \multicolumn{5}{|l|}{ Coronary angiogram } \\
\hline Left main disease & $27(12.7)$ & $5(7.8)$ & $32(11.6)$ & $0.281^{\dagger}$ \\
\hline Single-vessel disease & $82(38.7)$ & $23(35.9)$ & $105(38.0)$ & $0.692^{\dagger}$ \\
\hline Two-vessel disease & $39(18.4)$ & $17(26.6)$ & $56(20.3)$ & $0.155^{\dagger}$ \\
\hline Three-vessel disease & $79(37.3)$ & $20(31.3)$ & $99(35.9)$ & $0.177^{\dagger}$ \\
\hline \multicolumn{5}{|l|}{ Discharge therapy } \\
\hline Beta blockers & $158(74.5)$ & $42(65.6)$ & $200(72.5)$ & $0.162^{\dagger}$ \\
\hline ACE-I/ARB & $132(62)$. & $44(68.8)$ & $176(63.8)$ & $0.344^{\dagger}$ \\
\hline ASA & 199 (93.9) & $60(93.8)$ & $259(93.8)$ & $0.973^{\dagger}$ \\
\hline P2Y12 inhibitors & $182(85.8)$ & $50(78.1)$ & $232(84.1)$ & $0.139^{\dagger}$ \\
\hline Statins & $196(92.5)$ & $56(87.5)$ & $252(91.3)$ & $0.218^{\dagger}$ \\
\hline \multicolumn{5}{|l|}{ Risk scores } \\
\hline GRACE 2.0 & $99.0(80.3-116.0)$ & $107.5(88.5-127.3)$ & $100.0(82.0-120.0)$ & $0.072^{*}$ \\
\hline ACEF & $1.1(0.9-1.3)$ & $1.3(0.9-1.4)$ & $1.2(0.9-1.3)$ & $0.002^{\ddagger}$ \\
\hline SYNTAX & $14.0(8.0-23.4)$ & $13.3(7.0-26.0)$ & $14.0(8.0-24.0)$ & $0.869^{\ddagger}$ \\
\hline Clinical SYNTAX & $14.8(8.6-26.9)$ & $15.1(8.4-31.0)$ & $14.9(8.6-27.7)$ & $0.462^{\ddagger}$ \\
\hline SYNTAX II PCI & $25.5(20.4-33.7)$ & $29.8(24.1-38.5)$ & $26.8(20.8-34.8)$ & $0.001^{*}$ \\
\hline SYNTAX \| CABG & $24.9(17.2-32.0)$ & $27.0(18.7-36.4)$ & $25.5(18.1-33.6)$ & $0.038^{*}$ \\
\hline
\end{tabular}

Data are expressed as number (percent) or median (interquartile range)

ACE-I—angiotensin converting enzyme inhibitors; ACEF—Age, Creatinine and Ejection Fraction risk score; ARB-angiotensin receptor blockers; ASA—acetylsalicylic acid; CABG — coronary artery bypass grafting; GRACE—Global Registry of Acute coronary events risk score; MACE—-major adverse cardiovascular events; PCI_percutaneous coronary intervention; SYNTAX-The Synergy Between Percutaneous Coronary Intervention with TAXUS and Cardiac Surgery risk score

*Student's T test; ${ }^{\dagger}$ Chi-square test; ${ }^{\ddagger}$ Mann-Whitney $\mathrm{U}$ test

model containing ACEF, GRACE and SYNTAX II PCI score, $A C E F$ proved to be the only significant independent predictor for MACE (HR 2.16, 95\% CI 1.36-3.44, $P=0.001)$. There was no significant association of treatment strategy with MACE (Table 3). The cumulative incidence of MACE was greater in the patients within the highest $A C E F$ tertile (score $>1.24$ ) and above the optimal $A C E F$ cut-off value (score $>1.19$ ), with approximately 10-months earlier MACE development during follow-up (38.2, 95\% CI 34.1-42.3 vs. 48.5, 95\% CI 46.051.0 months, $P<0.001$; and $38.4,95 \%$ CI $34.6-42.2$ vs. 49.1, 95\% CI 46.5-51.6 months, $P<0.001$, respectively) (Fig. 1).

The ACEF and SYNTAX II PCI risk score had the best discriminatory accuracy to predict MACE, with an AUC value of $0.630(P=0.002)$ and $0.626(P=0.002)$, respectively. The sensitivity and specificity were $60.9 \%$ and $67.9 \%$, respectively for the value of the $A C E F$ score of 1.19. Overall performance of GRACE, SYNTAX, Clinical SYNTAX and SYNTAX II CABG was worse with AUC values of $0.596(P=0.020), 0.507(P=0.869), 0.530$
$(P=0.462)$ and $0.582(P=0.058)$, respectively. The best absolute AUC value of $0.680(P<0.001)$, which improved overall accuracy, showed the constructed model in which $A C E F$, female gender and atrial fibrillation were combined (Fig. 2). A post-hoc analysis of the computed predicted probabilities and AUC values revealed a relatively greater contribution of the female gender in the model (Additional file 2: Tables S4 and S5). Furthermore, a post-hoc ROC comparison revealed that AUC value of the constructed model does not differ statistically significantly from ACEF $(P=0.141)$ and SYNTAX II PCI $(P=0.123)$, while AUC value of $A C E F$ does not differ statistically significantly from GRACE $(P=0.342)$ and $S Y N$ TAX II PCI $(P=0.912)$, but are both better in comparison to other risk scores $(P<0.05)$. The Hosmer-Lemeshow test proved adequate calibration for predicting rates of MACE: ACEF ( $\mathrm{\chi} 2$ 15.77, $P=0.056)$, SYNTAX II PCI ( $\mathrm{\chi} 2$ 5.01, $P=0.757$, GRACE ( $\chi^{2}$ 6.63, $\left.P=0.578\right)$, SYNTAX $\left(\chi^{2}\right.$ 5.37, $P=0.615)$, Clinical SYNTAX ( $\chi 2$ 5.82, $P=0.667)$, SYNTAX II CABG ( $\left.\chi^{2} 12.98, P=0.113\right)$ and constructed model $\left(x^{2} 17,11, P=0.059\right)$. 
Table 3 Predictors of MACE

A. Individual variables (w/o risk scores)

\begin{tabular}{|c|c|c|c|c|}
\hline \multirow[t]{2}{*}{ Variables } & \multicolumn{2}{|c|}{ Univariate Cox analysis } & \multicolumn{2}{|c|}{ Multivariate Cox analysis } \\
\hline & $\mathrm{HR}(95 \% \mathrm{Cl})$ & $P$ value & $\mathrm{HR}(95 \% \mathrm{Cl})$ & $P$ value \\
\hline Age & $1.04(1.01-1.06)$ & 0.004 & $1.03(1.00-1.05)$ & 0.023 \\
\hline BMI & $0.93(0.87-0.99)$ & 0.030 & $\mathrm{~ns}^{\ddagger}$ & \\
\hline eGFR & $0.99(0.98-1.00)$ & 0.009 & $\mathrm{~ns}^{\ddagger}$ & \\
\hline Female gender & $1.86(1.12-3.09)$ & 0.016 & $1.67(1.00-2.79)$ & 0.051 \\
\hline Atrial fibrillation & $2.24(1.17-4.29)$ & 0.015 & $2.02(1.04-3.89)$ & 0.037 \\
\hline Previous MI & $1.75(0.92-3.35)$ & 0.091 & $\mathrm{~ns}^{\ddagger}$ & \\
\hline $\begin{array}{l}\text { Conservative treatment vs. revasculari- } \\
\text { zation }\end{array}$ & $1.53(0.90-2.60)$ & 0.114 & $n / a^{\S}$ & \\
\hline
\end{tabular}

\section{B. Risk scores}

\begin{tabular}{|c|c|c|c|c|}
\hline \multirow[t]{2}{*}{ Variables } & \multicolumn{2}{|c|}{ Univariate Cox analysis } & \multicolumn{2}{|c|}{ Multivariate Cox analysis } \\
\hline & $\mathrm{HR}(95 \% \mathrm{Cl})$ & $P$ value & $\mathrm{HR}(95 \% \mathrm{Cl})$ & $P$ value \\
\hline \multirow[t]{2}{*}{ GRACE } & $1.01(1.00-1.02)^{*}$ & 0.088 & $0.97(0.86-1.10)^{\|}$ & 0.664 \\
\hline & $1.09(0.99-1.19)^{\dagger}$ & 0.076 & & \\
\hline ACEF & $2.16(1.36-3.44)^{*}$ & 0.001 & $2.16(1.36-3.44)^{\mathbb{q}}$ & 0.001 \\
\hline \multirow[t]{2}{*}{ SYNTAX } & $1.00(0.98-1.03)^{*}$ & 0.880 & $\mathrm{n} / \mathrm{a}^{\S}$ & \\
\hline & $0.99(0.79-1.25)^{\dagger}$ & 0.948 & & \\
\hline \multirow[t]{2}{*}{ Clinical SYNTAX } & $1.01(1.00-1.03)^{*}$ & 0.063 & $\mathrm{n} / \mathrm{a}^{\S}$ & \\
\hline & $1.14(0.99-1.29)^{\dagger}$ & 0.059 & & \\
\hline \multirow[t]{2}{*}{ SYNTAX II PCI } & $1.04(1.01-1.06)^{*}$ & 0.001 & $1.22(0.88-1.69)^{\|}$ & 0.237 \\
\hline & $1.37(1.11-1.79)^{\dagger}$ & 0.004 & & \\
\hline \multirow[t]{2}{*}{ SYNTAX $\| C A B G$} & $1.02(1.00-1.04)^{*}$ & 0.040 & $\mathrm{n} / \mathrm{a}^{\S}$ & \\
\hline & $1.22(1.00-1.50)^{\dagger}$ & 0.053 & & \\
\hline
\end{tabular}

ACEF_-age, creatinine and ejection fraction risk score; BMI—body mass index; CABG—coronary artery bypass graft; eGFR—estimated glomerular filtration rate; GRACE - Global Registry of Acute coronary events risk score; LVEF_left ventricular ejecton fraction; MACE_-major adverse cardiovascular events; MI_-myocardial infarction; $\mathrm{PCl}$ - percutaneous coronary intervention; SYNTAX-The Synergy Between Percutaneous Coronary Intervention with TAXUS and Cardiac Surgery risk score

* HR value corresponds to each 1 unit increase/decrease of each score

+ HR value corresponds to each 10 unit increase/decrease of each score

‡ ns indicates variables which were not held in the model due to statistical insignificance (stepwise forward algorithm)

$\S \mathrm{n} / \mathrm{a}$ indicates variables which were a priori not included in the multivariate analysis due to $P>0.1$ and/or multicollinearity

$\|$ The intermediate step of the multivariate analysis (stepwise forward algorithm)

^ The final step of the multivariate analysis (stepwise forward algorithm)
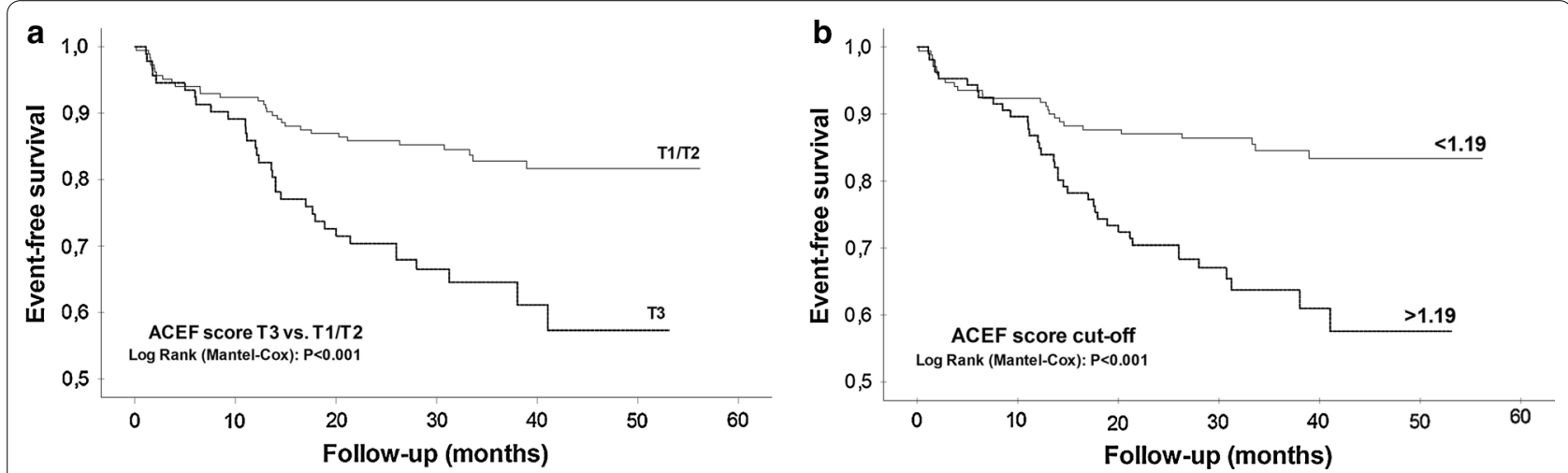

Fig. 1 Event-free survival for MACE: a ACEF tertiles (T1/T2 vs. T3); b Optimal prognostic cut-off value*. T1—ACEF risk score first tertile (values $<1.00)$; T2-ACEF risk score second tertile (values 1.00-1.24); T3-ACEF risk score third tertile (values $>1.24$ ); ${ }^{*}$ determined by Youden index $(<1.19 \mathrm{vs} .>1.19)$ 


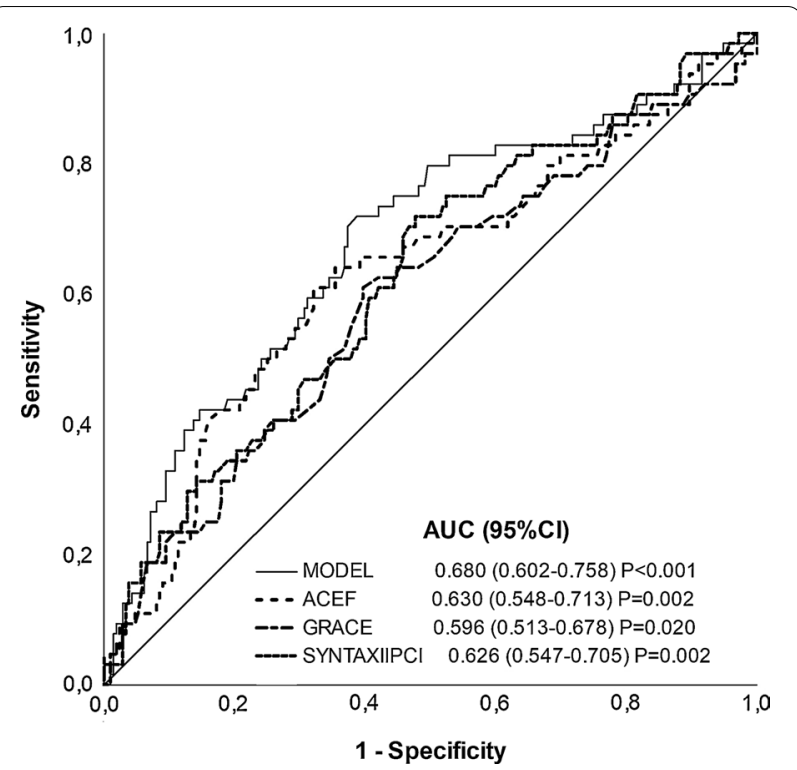

Fig. 2 Receiver operating characteristic of predicting MACE for MODEL *, ACEF, SYNTAX $\| \mathrm{PCl}$ and GRACE score. *Computation of ACEF, female gender and atrial fibrillation; ${ }^{\dagger} P$ values represent the significance relatively to the non-informative $\mathrm{ROC}$ curve (AUC $=0.5$ ) and were tested using a SPSS algorithm based on methodology of Hanley and MCNeil; ACEF-Age, Creatinine and Ejection Fraction risk score; GRACE-Global Registry of Acute coronary events risk score; LVEF_left ventricular ejection fraction; $\mathrm{PCl}$ — percutaneous coronary intervention; SYNTAX - The Synergy Between Percutaneous Coronary Intervention with TAXUS and Cardiac Surgery risk score

\section{Discussion}

The risk stratification of NSTE-ACS patients represents a crucial role in everyday clinical settings. However, head-to-head comprehensive studies comparing the most utilized available risk scores in NSTE-ACS patients undergoing all-treatment strategies (PCI, CABG or conservatively), with long-term follow-up, are lacking. To our knowledge, only Stahli et al. and Palmerini et al. have questioned the role of several risk scores on 1-year outcomes in the NSTE-ACS patients managed by revascularization methods $[10,19]$. This study demonstrates a comparison of GRACE 2.0, ACEF, SYNTAX, Clinical SYNTAX, SYNTAX II PCI and SYNTAX II $C A B G$ in NSTE-ACS patients undergoing all-treatment strategies, over a remarkably long-term follow-up (up to 56 months).

This study has established several key findings. First, patients who developed MACE had statistically significantly increased $A C E F$ and SYNTAX II scores. Second, $A C E F$ and SYNTAX II PCI proved the best discriminatory accuracy in predicting MACE, with an acceptable sensitivity and specificity ratio. Finally, the cumulative incidence of MACE was statistically significantly greater in the patients within the highest $A C E F$ tertile and above the optimal $A C E F$ cut-off value with approximately 10-months earlier MACE development.

$A C E F$ risk score incorporates only three variables, thereby representing one of the simplest scores in terms of assessment [10, 15]. Risk assessment using an angiography-related risk scores was proposed as well, with SYNTAX score being the first fully-based on angiographic features $[11,12]$. However, the absence of clinical variables has contributed to substantial limitations of the SYNTAX score $[11,13]$. To overcome these limitations Clinical SYNTAX risk score has been developed by multiplying values of modified ACEF and SYNTAX score [13]. Further improvement was the inclusion of additional clinical and anatomic variables resulting in the SYNTAX II score [14]. Although these scores were developed to estimate patient prognosis and to provide optimal patient-oriented treatment, they are still largely underused [20]. Some of the main concerns are their development and validation in different clinical settings, across the entire spectrum of both stable and unstable patients, managed with different treatment strategies [21-24]. Furthermore, their prognostic strength was evaluated in different time-frames, from in-hospital outcomes to short-term and long-term follow-up $[6,19]$. Finally, the complexity of risk scores aggravates everyday clinical usage indicating the importance of simple clinical scores [20].

Therefore, features of $A C E F$ like simplicity of application, easily accessible components, and time-sparing properties offer potential benefits for the clinicians [15] making these results encouraging. Previous studies have reported a risk stratification role of $A C E F$ in different clinical settings [25-27], but research on ACS patients have been mostly based on a heterogeneous sample of PCI-treated patients $[23,24]$. The findings of good predictive value of SYNTAX II PCI score in this population is likewise reassuring given that it's clinical attributes (age, creatinine clearance and left ventricular ejection fraction) are in fact the components of $A C E F$ score [16].

Large Acute Catheterization and Urgent Intervention Triage Strategy (ACUITY) trial compared several risk scores in the prediction of 1-year clinical adverse outcomes amongst NSTE-ACS population treated with PCI. They established the best predictive accuracy for combined clinical and angiographic scores (AUC 0.60) which is followed by purely angiographic SYNTAX score (AUC 0.59 ), but only poor to modest discrimination strength of fully-clinical ACEF (AUC 0.52) and GRACE (AUC $0.52)$ scores [19]. Similar findings were reported in the LEADERS Trial amongst all-comers population undergoing PCI which showed AUC values of 0.62 and 0.58 , for Clinical SYNTAX and ACEF risk scores, respectively [24]. On the contrary, the present study has shown the 
best discriminatory accuracy for MACE prediction with $A C E F$ and SYNTAX II PCI scores, while GRACE, SYN$T A X$ and Clinical SYNTAX had poorer overall performance. In general, this study exhibited higher absolute MACE-related AUC values for $A C E F$, similar for GRACE, but lower for SYNTAX and Clinical SYNTAX scores, in comparison to the ACUITY trial [19]. The poor overall performance of the GRACE score in this long-term study is consistent with previous studies and could be explained by its initial development for the prediction of short-term events $[28,29]$. A similar study by Stahli et al. compared the predictive role of $A C E F$ and GRACE scores on 30-day and 1-year outcomes in the total cohort of ACS patients treated with PCI or CABG. Complementary to the present study, they showed a significant independent association of the highest $A C E F$ group with 1-year MACCE (HR 3.75, 95\% CI 2.56-5.49) [10]. Furthermore, the data from the Korean Acute Myocardial Infarction Registry comprising a total ACS cohort undergoing PCI showed that the ACEF score was an independent predictor of 1-year mortality with a robust AUC of 0.79 [30]. However, as with a study by Stahli et al., more than half of a study sample was encompassed by STEMI patients which are known to have less benefit from risk stratification (61.3\% and 53.6\%, respectively) [10, 30]. Additionally, they only enrolled patients referred for coronary revascularization, with CABG rate being presumably low $(\sim 4 \%)$. The exclusion of pharmacologically- and small proportion of surgically-treated patients in these studies represents a possible limitation for applying these results to the general NSTE-ACS population $[10,19,30]$.

Several factors could explain the findings of respectable AUC values of purely clinical risk score, while previous studies have demonstrated the predominance of combined clinical and angiographic risk scores [19, 24]. Firstly, a different study population which encompassed only NSTE-ACS patients which were treated with all management strategies could be less affected by the angiographic findings. Furthermore, longer follow-up in the present study could diminish the influence of angiographic differences from SYNTAX-related scores or acute setting parameters which are components of GRACE score (cardiac arrest on admission; Killip class; abnormal cardiac enzymes; ECG changes). It is possible that age, kidney function and cardiac capacity, even though determined by crude parameters, have similar influence on short-term and long-term outcomes, while the inclusion of other parameters have more impact on the treatment strategy and short-term prognosis. Finally, most previous similar studies have used a composite outcome consisting of all-cause mortality or included major bleeding which were not assessed by this study. This fact could also play a certain role in the discriminatory power of the risk scores. Nevertheless, these findings indicate that $A C E F$ could possibly performs good enough in comparison to other complex risk scores and may serve as a fast and user-friendly tool to stratify NSTE-ACS patients.

The median follow-up of the present study was 33 months with a maximal period of up to 56 months. None of the available studies provided a similar followup period and insights into the role of $A C E F$ in the prediction of such extended long-term outcomes. While the aforementioned studies have mostly provided insights for the 1-year outcomes, only Chichareon et al. have reported longer follow-up in all-comers in the GLOBAL LEADERS study [23].

Consistent with previous studies, the present analysis has revealed that female NSTE-ACS patients were more likely to develop adverse outcomes. Gender disparities in outcomes after MI have been reported in several studies but surpass the horizons of this paper [31, 32]. Nevertheless, a modest improvement in accuracy of $A C E F$ was obtained with the addition of female gender and atrial fibrillation in the computed model in this study (AUC 0.680 ). These findings are not surprising since atrial fibrillation is a well-established negative prognostic factor [33].

As with the other studies, this study has several limitations. A small sample size with a relatively low incidence of end-points does not allow for additional sub-analyses, while the statistically significant low absolute values of $\mathrm{C}$-statistics require careful interpretation of the true clinical significance. The composite outcome MACE included cardiac death, nonfatal MI, ischemic stroke, and urgent coronary revascularization which may impede its comparison to other studies. Furthermore, this study encompassed a relatively low-risk population of NSTEACS patients with a large heterogeneity in follow-up which is reflected by basal patient characteristics and aggravates its extrapolation to the total NSTE-ACS population. Similarly, the results are not applicable to patients with active malignancy or previous CABG, as they were excluded. Moreover, the study was conducted before the era of high-sensitivity cardiac troponin assays, and only NSTE-ACS patients who underwent coronary angiography were included. SYNTAX II scores were used in the clinical setting in which they were not validated, i.e. $S Y N$ TAX II PCI in patients undergoing CABG or conservative management and SYNTAX II CABG in patients undergoing PCI or conservative management [14]. Finally, $A C E F$ categories in this study were created using original score tertiles which impede result comparisons and inter-analyses with some other studies.

In conclusion, a simple clinical risk score $A C E F$ exhibited better discrimination compared to other complex risk scores, in NSTE-ACS patients undergoing 
all-treatment strategies over the long-term follow-up. Therefore, $A C E F$ could possibly represent a fast and userfriendly tool to stratify NSTE-ACS patients. Future longterm prospective studies are necessary to strengthen this association and determine other clinical elements which might improve prognostic strength in this patient population.

\section{Supplementary information}

The online version contains supplementary material available at https://doi. org/10.1186/s12872-020-01841-2.

Additional file 1. Fig. S1: Flow diagram of the study design.

Additional file 2. Table S1: Overview of the first risk score validation in the setting of ACS or PCI. Table S2: Description of risk scores. Table S3 Association of risk scores with occurrence of major adverse cardiovascular events. Table S4: Comparison of predicted probabilities of ACEF and different computed models (derived from bivariate logistic regression). Table S5: ROC curve analysis.

\section{Abbreviations}

ACEF: Age, creatinine, ejection fraction score; ACS: Acute coronary syndrome; AUC: Area under the curve; CABG: Coronary artery bypass graft; GRACE: Global registry for acute coronary events score; MACE: Major adverse cardiac event; MI: Myocardial infarction; NSTE-ACS: Non-ST-elevation acute coronary syndrome; NSTEMI: Non-ST-segment elevation myocardial infarction; PCI: Percutaneous coronary intervention; ROC: Receiver operating characteristic; STEMI: ST-segment elevation myocardial infarction; SYNTAX: The Synergy Between Percutaneous Coronary Intervention with TAXUS and Cardiac Surgery score; UA: Unstable Angina.

\section{Acknowledgements}

None.

\section{Authors' contributions}

Substantial contributions to the conception and design of the study: IK, NC, FR, AM, MV; acquisition of data: IK, NC, FR, OP; analysis and interpretation of data: IK, VČ, AM, MV; drafting the article or revising it critically for important intellectual content: IK, AM, OP, MV; final approval of the version to be submitted: IK, NC, FR, VČ, OP, AM, MV; have agreed both to be personally accountable for the author's own contribution: IK, NC, FR, VČ, OP, AM, MV. All authors read and approved the final version of the article, including the authorship list.

\section{Funding}

This research received no grant support or other external funding.

\section{Availability of data and materials}

We disclose any restrictions on the availability of data, materials and associated protocols. The datasets used and/or analysed during the current study are available from the corresponding author on reasonable request.

\section{Ethics approval and consent to participate}

This clinical investigation has been performed in accordance with the ethical standards of the Declaration of Helsinki and its later amendments. The study protocol was approved by the Ethical Committee of the University Hospital of Split, Croatia (No. 2181-147-01/06). All the participants included in the study provided formal written informed consent for coronary angiography and informed verbal consent for the use of relevant medical data. All participants were informed about the goal and course of this study.

\section{Consent for publication}

Not applicable.

\section{Competing interests}

The authors declare that they have no competing interests.

\section{Author details}

${ }^{1}$ Department of Cardiology, University Hospital of Split, Split, Croatia. ${ }^{2}$ University Department of Health Studies, University of Split, Split, Croatia. ${ }^{3}$ Department of Public Health, School of Medicine, University of Split, Split, Croatia.

${ }^{4}$ Department of Cardiology, Sestre Milosrdnice University Hospital Center, Vinogradska cesta 29, 10000 Zagreb, Croatia. ${ }^{5}$ University of Zagreb School of Medicine, Zagreb, Croatia.

Received: 23 May 2020 Accepted: 27 December 2020

Published online: 03 February 2021

References

1. Roger VL, Weston SA, Gerber Y, Killian JM, Dunlay SM, Jaffe AS, Bell MR, Kors J, Yawn BP, Jacobsen SJ. Trends in incidence, severity, and outcome of hospitalized myocardial infarction. Circulation. 2010;121(7):863-9.

2. Yeh RW, Sidney S, Chandra M, Sorel M, Selby JV, Go AS. Population trends in the incidence and outcomes of acute myocardial infarction. N Engl J Med. 2010;362(23):2155-65.

3. Amsterdam EA, Wenger NK, Brindis RG, Casey DE, Ganiats TG, Holmes DR, Jaffe AS, Jneid H, Kelly RF, Kontos MC, et al. 2014 AHA/ACC guideline for the management of patients with non-ST-elevation acute coronary syndromes. J Am Coll Cardiol. 2014;64(24):e139.

4. Collet J-P, Thiele H, Barbato E, Barthélémy O, Bauersachs J, Bhatt DL, Dendale P, Dorobantu M, Edvardsen T, Folliguet T et al: 2020 ESC Guidelines for the management of acute coronary syndromes in patients presenting without persistent ST-segment elevation: The Task Force for the management of acute coronary syndromes in patients presenting without persistent ST-segment elevation of the European Society of Cardiology (ESC). European Heart Journal 2020.

5. Piatek L, Janion-Sadowska A, Piatek K, Zandecki L, Zabojszcz M, Siudak Z, Sadowski M: Long-term clinical outcomes in patients with unstable angina undergoing percutaneous coronary interventions in a contemporary registry data from Poland. Coronary Artery Disease 2019.

6. Steg PG, Goldberg RJ, Gore JM, Fox KA, Eagle KA, Flather MD, Sadiq I, Kasper R, Rushton-Mellor SK, Anderson FA. Baseline characteristics, management practices, and in-hospital outcomes of patients hospitalized with acute coronary syndromes in the Global Registry of Acute Coronary Events (GRACE). Am J Cardiol. 2002:90(4):358-63.

7. Rodriguez F, Mahaffey KW. Management of patients with NSTE-ACS: a comparison of the recent AHA/ACC and ESC guidelines. J Am Coll Cardiol. 2016;68(3):313-21.

8. Fox KA, Dabbous OH, Goldberg RJ, Pieper KS, Eagle KA, Van de Werf F, Avezum A, Goodman SG, Flather MD, Anderson FA, et al. Prediction of risk of death and myocardial infarction in the six months after presentation with acute coronary syndrome: prospective multinational observational study (GRACE). BMJ. 2006;333(7578):1091.

9. Granger CB, Goldberg RJ, Dabbous O, Pieper KS, Eagle KA, Cannon CP, Van de Werf F, Avezum Á, Goodman SG, Flather MD, et al. Predictors of hospital mortality in the global registry of acute coronary events. Arch Intern Med. 2003;163(19):2345-53.

10. Stähli BE, Wischnewsky MB, Jakob P, Klingenberg R, Obeid S, Heg D, Räber $L$, Windecker S, Roffi M, Mach F, et al. Predictive value of the age, creatinine, and ejection fraction (ACEF) score in patients with acute coronary syndromes. Int J Cardiol. 2018;270:7-13.

11. Sianos G, Morel MA, Kappetein AP, Morice MC, Colombo A, Dawkins K, van den Brand M, Van Dyck N, Russell ME, Mohr FW, et al. The SYNTAX Score: an angiographic tool grading the complexity of coronary artery disease. Eurolntervention. 2005;1(2):219-27.

12. Serruys PW, Morice M-C, Kappetein AP, Colombo A, Holmes DR, Mack MJ, Ståhle E, Feldman TE, van den Brand M, Bass EJ, et al. Percutaneous coronary intervention versus coronary-artery bypass grafting for severe coronary artery disease. N Engl J Med. 2009;360(10):961-72.

13. Garg S, Sarno G, Garcia-Garcia HM, Girasis C, Wykrzykowska J, Dawkins KD Serruys PW. A new tool for the risk stratification of patients with complex coronary artery disease: the Clinical SYNTAX Score. Circul Cardiovasc Interv. 2010;3(4):317-26.

14. Farooq V, van Klaveren D, Steyerberg EW, Meliga E, Vergouwe Y, Chieffo A, Kappetein AP, Colombo A, Holmes DR Jr, Mack M, et al. Anatomical and clinical characteristics to guide decision making between coronary artery 
bypass surgery and percutaneous coronary intervention for individual patients: development and validation of SYNTAX score II. Lancet (London). 2013;381(9867):639-50.

15. Ranucci M, Castelvecchio S, Menicanti L, Frigiola A, Pelissero G. Risk of assessing mortality risk in elective cardiac operations: age, creatinine, ejection fraction, and the law of parsimony. Circulation. 2009;119(24):3053-61

16. Wang G, Wang C, Zhang Y, Wang P, Ran C, Zhao L, Han L. Usefulness of the SYNTAX score II to predict 1-year outcome in patients with primary percutaneous coronary intervention. Coron Artery Dis. 2016;27(6):483-9.

17. Global Registry of Acute Coronary Syndrome (GRACE). https://www. outcomes-umassmed.org/GRACE/grace_risk_table.aspx.

18. Hanley JA, MCNeil BJ. The meaning and use of the area under a receiver operating characteristic (ROC) curve. Radiology. 1982;143(1):29-36.

19. Palmerini T, Caixeta A, Genereux P, Cristea E, Lansky A, Mehran R, Dangas G, Lazar D, Sanchez R, Fahy M et al: Comparison of clinical and angiographic prognostic risk scores in patients with acute coronary syndromes: Analysis from the Acute Catheterization and Urgent Intervention Triage StrategY (ACUITY) trial. Am Heart J 2012;163(3):383-391, 391.e381-385.

20. Chan Pin Yin D, Azzahhafi J, James S. Risk assessment using risk scores in patients with acute coronary syndrome. J Clin Med. 2020;9:9.

21. Gao S, Liu Q, Ding X, Chen H, Zhao X, Li H. Predictive value of the combination of age, creatinine, and ejection fraction score and diabetes in patients with ST-segment elevation myocardial infarction undergoing percutaneous coronary intervention. Coron Artery Dis. 2020;31(2):109-17.

22. Wang L, Huang G, Peng Q, Duan C, Dai Y, Shao S, Zhong WW, Yu D, Chen $J$, Xue L, et al. Risk predictive ability of ACEF score for infection in patients with ST-segment elevation myocardial infarction undergoing percutaneous coronary intervention. Eur J Prevent Cardiol. 2020;27(2):220-2.

23. Chichareon P, Modolo R, van Klaveren D, Takahashi K, Kogame N, Chang CC, Katagiri Y, Tomaniak M, Asano T, Spitzer E, et al. Predictive ability of ACEF and ACEF II score in patients undergoing percutaneous coronary intervention in the GLOBAL LEADERS study. Int J Cardiol. 2019;286:43-50.

24. Wykrzykowska JJ, Garg S, Onuma Y, de Vries T, Goedhart D, Morel MA, van Es GA, Buszman P, Linke A, Ischinger T, et al. Value of age, creatinine, and ejection fraction (ACEF score) in assessing risk in patients undergoing percutaneous coronary interventions in the "All-Comers" LEADERS trial. Circul Cardiovas Interv. 2011;4(1):47-56.

25. Sullivan PG, Wallach JD, loannidis JP. Meta-analysis comparing established risk prediction models (EuroSCORE II, STS Score, and ACEF score) for perioperative mortality during cardiac surgery. Am J Cardiol. 2016;118(10):1574-82.

26. Ni Z, Liang Y, Xie N, Liu J, Sun G, Chen S, Ye J, He Y, Guo W, Tan N, et al. Simple pre-procedure risk stratification tool for contrast-induced nephropathy. J Thor Dis. 2019;11(4):1597-610.

27. Tsai TY, Tsai FC, Fan PC, Chang CH, Lin CY, Chang WW, Lee SY, Hsu HH, Tian YC, Fang JT, et al. Application of the age, creatinine, and left ventricular ejection fraction score for patients on extracorporeal membrane oxygenation. Artif Organ. 2017;41(2):146-52.

28. Persampieri S, Castini D, Valli F, Sabatelli L, Carugo S. Additional predictive value of C-reactive protein to GRACE score in patients with acute coronary syndrome. Eur J Intern Med. 2019;69:e1-2.

29. Eagle KA, Lim MJ, Dabbous OH, Pieper KS, Goldberg RJ, Van de Werf F, Goodman SG, Granger CB, Steg PG, Gore JM, et al. A validated prediction model for all forms of acute coronary syndrome: estimating the risk of 6-month postdischarge death in an international registry. JAMA. 2004;291(22):2727-33.

30. Lee $J H$, Bae MH, Yang DH, Park HS, Cho Y, Jeong MH, Kim YJ, Kim KS, Hur $\mathrm{SH}$, Seong IW, et al. Prognostic value of the age, creatinine, and ejection fraction score for 1-year mortality in 30-day survivors who underwent percutaneous coronary intervention after acute myocardial infarction. Am J Cardiol. 2015;115(9):1167-73.

31. Vrsalovic M, Pintaric H, Babic Z, Pavlov M, Vrsalovic Presecki A, Getaldic B, Vrkic N, Nikolic Heitzler V. Impact of admission anemia, C-reactive protein and mean platelet volume on short term mortality in patients with acute ST-elevation myocardial infarction treated with primary angioplasty. Clin Biochem. 2012:45(16-17):1506-9.

32. Lundblad D, Holmgren L, Jansson J-H, Näslund U, Eliasson M. Gender differences in trends of acute myocardial infarction events: the Northern Sweden MONICA study 1985-2004. BMC Cardiovasc Disord 2008:8:17-17.

33. Vrsalovic M, Presecki AV. Atrial fibrillation and risk of cardiovascular events and mortality in patients with symptomatic peripheral artery disease: a meta-analysis of prospective studies. Clin Cardiol. 2017;40(12):1231-5.

\section{Publisher's Note}

Springer Nature remains neutral with regard to jurisdictional claims in published maps and institutional affiliations.
Ready to submit your research? Choose BMC and benefit from:

- fast, convenient online submission

- thorough peer review by experienced researchers in your field

- rapid publication on acceptance

- support for research data, including large and complex data types

- gold Open Access which fosters wider collaboration and increased citations

- maximum visibility for your research: over $100 \mathrm{M}$ website views per year

At BMC, research is always in progress.

Learn more biomedcentral.com/submissions 